\title{
Switchable nanometer contacts: Ultrathin Ag nanostructures on $\mathrm{Si}(100)$
}

G. Gardinowski, J. Schmeidel, H. Pfnür, T. Block, and C. Tegenkamp

Citation: Appl. Phys. Lett. 89, 063120 (2006);

View online: https://doi.org/10.1063/1.2336650

View Table of Contents: http://aip.scitation.org/toc/apl/89/6

Published by the American Institute of Physics

\section{Articles you may be interested in}

Electronic and chemical properties of the $\mathrm{c}-\mathrm{Si} / \mathrm{Al}_{2} \mathrm{O}_{3}$ interface

Journal of Applied Physics 109, 113701 (2011); 10.1063/1.3587227

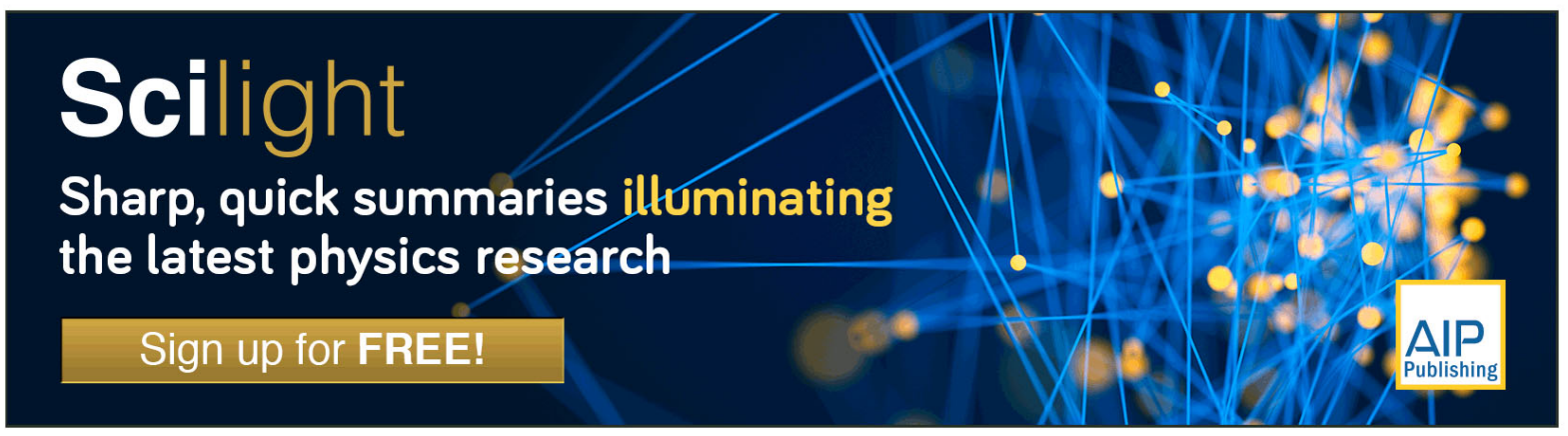




\title{
Switchable nanometer contacts: Ultrathin Ag nanostructures on $\mathrm{Si}(100)$
}

\author{
G. Gardinowski, J. Schmeidel, H. Pfnür, T. Block, and C. Tegenkamp ${ }^{\text {a) }}$ \\ Institut für Festkörperphysik, Universität Hannover, Appelstraße 2, D-30167 Hannover, Germany
}

(Received 31 March 2006; accepted 8 July 2006; published online 10 August 2006)

\begin{abstract}
The fabrication and characterization of metallic nanometer-sized gaps suitable for conductivity measurements of single molecules were studied. Controlled gap formation by electromigration (EM) is demonstrated in contiguous and ultrathin $\mathrm{Ag}$ structures wetting the $\mathrm{Si}(100)$ substrate. The gaps obtained are in the range of nanometers or even subnanometers, as revealed by lateral conductivity measurements and scanning tunneling microscopy carried out under ultrahigh vacuum conditions. Annealing to $300 \mathrm{~K}$ closes the gap by enabling surface diffusion of $\mathrm{Ag}$, and another cycle of opening by EM at $80 \mathrm{~K}$ can be performed. The functionality of the contacts is demonstrated by insertion of ferrocenedithiol molecules into the gap. (c) 2006 American Institute of Physics.
\end{abstract}

[DOI: $10.1063 / 1.2336650$ ]

The ultimate limit of nanoelectronics is the use of single molecules as functional units. One of the key techniques to reach this goal is the generation of reliable contacts with separations in the range of only $1 \mathrm{~nm}$, which allow testing of the conductive properties of single molecules and control of their functionalities. This goal was the reason for several successful attempts for the generation of nanocontacts for single molecules in the recent past. ${ }^{1-6}$ Since the required gap size is an order of magnitude below those structural widths feasible by present-day lithography techniques, several alternative approaches were reported. They combine lithographically produced structures with electromigration processes to thin and interrupt the contact ${ }^{1}$ or use proximity effects, ${ }^{6}$ mechanically controllable break junctions, ${ }^{2-4}$ selective etching, ${ }^{5}$ or the recently developed conducting DNA networks. ${ }^{7}$

All these nanocontacts still have the disadvantage that their local structure cannot be controlled. However, the local structure bonding, the molecule to the metallic leads, crucially determines the conductive properties of these quantum mechanical objects in case of strong coupling because molecule and leads must be considered as an entity. This was demonstrated by several recent calculations. ${ }^{8,9}$ Therefore, the control of the complete atomic arrangement in the vicinity of the gap and of the contact area to the molecule on the atomic scale is necessary in order to reach a more complete understanding of molecular switching properties. We show in this letter that we are able to produce not only structures with gaps in the range between $5 \mathrm{~nm}$ and below $1 \mathrm{~nm}$, but that these structures are thin enough and laterally open so that they are completely accessible to a scanning tunneling microscope (STM) for local control. Thus they fulfill the requirements just mentioned.

Silver as metal was chosen because of its higher chemical flexibility compared to gold, and because Ag grows epitaxially on $\mathrm{Si}(100)$ (Ref. 10) so that contiguous films are obtained for Ag layers that are less than $10 \mathrm{ML}$ thick, if they are grown at temperatures around $100 \mathrm{~K} .{ }^{10}$ The low-doped silicon substrates $(1000 \Omega \mathrm{cm}$ at $300 \mathrm{~K})$ are insulating at temperatures below $150 \mathrm{~K}$. In addition, for $\mathrm{Ag}$ as an adsorbate, band bending effects cause a depletion of the space charge carriers ${ }^{11}$ so that $\mathrm{Ag}$ is well isolated from the Si bulk

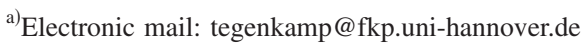

under our experimental conditions below room temperature.

We started out writing the nanostructures by electron beam lithography (EBL)with a JEOL 5900 scanning electron microscope (SEM) in combination with a Raith lithography system into a polymethylmethacrylate (PMMA)/LOR3B resist structure, which was spin coated at a thickness of 120 and $370 \mathrm{~nm}$, respectively, onto the $\mathrm{Si}(100)$ wafer [see Fig. 1(a)]. The wafer was cleaned before by $\mathrm{O}_{2}$-plasma etching. An electron dose of $180 \mu \mathrm{C} / \mathrm{cm}^{2}(\mathrm{HV}=30 \mathrm{keV})$ was sufficient to depolymerize the resist so that after development (AZ625) and etching, followed by a HF dip, a hydrogen passivated $\mathrm{Si}(100)$ surface within the written structure is left behind. After development the structure in the LOR3B layer is laterally larger than the openings in the PMMA layer. This effect was used to fabricate a nanobridge of PMMA, about $250 \mathrm{~nm}$ wide, above the $\mathrm{Si}(100)$ surface which was used as a shadow mask during the Ag evaporation [Fig. 1(b)]. Immediately after the HF dip the sample was introduced into UHV and cooled to $100 \mathrm{~K}$. At this temperature Ag was evaporated, and the amount controlled by a microbalance. The variation of the angle of incidence of the Ag flux allowed us to fabricate vertically structured Ag wires [Fig. 1(c)]. A schematic of this process is shown in Fig. 2. After optimization of the form of the nanobridge in order to minimize current and temperature gradients, and after removal of the restist, a freestanding three-dimensional Ag structure was obtained that we could further thin and finally break reproducibly at the narrowest point by electromigration (see below). A SEM image of a final structure at the typical size obtained after

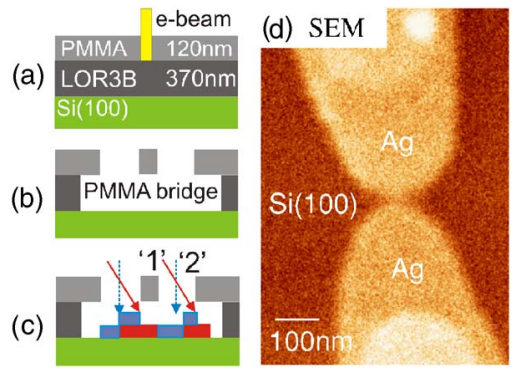

FIG. 1. (Color online) Scheme for fabricating a nanostructure by EBL using PMMA as a nanomask in the center of the structure. The SEM graph shows the structure after removal of the resists. The contrast is caused by the different $\mathrm{Ag}$ thicknesses within the nanostructure. For details see text. 

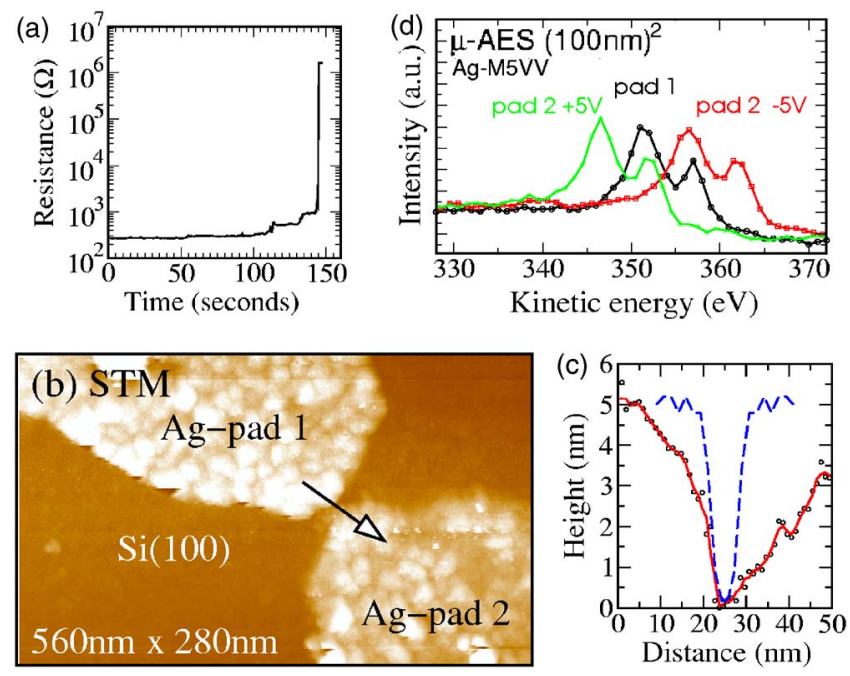

FIG. 2. (Color online) (a) Change in resistance of a Ag nanostructure vs time. Electromigration (EM) leads to destruction of the initially continuous wire. The applied constant voltage was $1 \mathrm{~V}$. (b) A characteristic STM image of the gap $(5 \mathrm{~nm})$ induced by the EM process. (c) Line scan taken along the arrow in (b). The dashed curve represents a worst-case approximation of the tip. (d) $\mu$-AES done on the two Ag pads close to the gap. The voltage drop of $\pm 5 \mathrm{~V}$ between the pads can be found again by shifting of the Ag peaks.

evaporation at only two different angles is shown in Fig. 1(d). It demonstrates that Ag structures that barely touch, but are still connected by a bridge several nanometers wide, can be made. The thickness of the Ag films have been varied between 2 and $15 \mathrm{~nm}$ for the first and second evaporation steps. Note that the $\mathrm{Ag}$ films produced are continuous even at a thickness of only $2 \mathrm{~nm}$, as tested both by atomic force microscopy and by STM. It seems that by our sample preparation we still form Ag layer that wet the Si surface. The direct proof is the closing of the gap formed by electromigration by annealing the structure to room temperature (see below). Since the minimal thickness of Ag close to the gap was below $10 \mathrm{ML}$, it is accessible for the STM, as we show below.

The gap itself was generated using a feedback-controlled electromigration (EM) technique. Reliable formation of gap widths of only a few angstroms required optimization of the wire form at the narrowest part, as mentioned, and cooling of the sample with $\ell \mathrm{N}_{2}$.

The resistance of these structures before EM was typically between 25 and $100 \Omega$. EM started to be effective for voltages around $1 \mathrm{~V}$, i.e., at a current density in the central part of the structure around $4 \times 10^{9} \mathrm{~A} / \mathrm{cm}^{2}$. Here we assume a typical cross section of approximately $50 \times 5 \mathrm{~nm}^{2}$, as judged from our SEM and STM images. Figure 2(a) shows the time evolution of the resistance during EM. Experimentally, the critical EM parameters were controlled by a selfwritten software, which increases the voltage gradually as a function of the change in current. Depending on the geometry of the initial structure, the final tunneling resistances obtained for different samples after EM vary between 0.2 and $10 \mathrm{M} \Omega$; i.e., the tunneling gaps are between $5 \AA$ and $5 \mathrm{~nm} .{ }^{12}$ The resistance of the $\operatorname{Si}(100)$ sample itself is at least an order of magnitude larger, as reference measurements have shown for nonprocessed $\mathrm{Si}(100)$ samples.

The absolute tunneling current depends on the contact geometry and the energy barriers in between. Therefore, we performed STM measurements to calibrate the gap sizes obtained from conductance measurements. A gap of around $5 \mathrm{~nm}$ after EM is shown in Fig. 2(b). The dashed curve in the line scan Fig. 2(c) represents the geometry of the tip, obtained from the edge of a large Ag cluster. Since the edge of this cluster was assumed to be vertical, we obtain an upper limit for the tip size. Correspondingly, $5 \mathrm{~nm}$ is an upper estimate of the gap size shown in Fig. 2, in accordance with a tunneling resistance of only $10 \mathrm{M} \Omega$.

Figure 2 shows several points worth noting: First, the tip reaches the bottom of the gap so that the whole gap structure can indeed be imaged. Second, the location of the gap coincides with the central part of the structure carrying the highest current density. Third, all other parts of the structure are intact, as can be seen, for instance, by the shifting of the Ag Auger peak after applying $\pm 5 \mathrm{~V}$ to the contacts [see Fig. 2(d)]. Fourth, the Ag surface is, despite the intense processing, chemically clean except for some carbon contamination (resist) of a few percent of a monolayer, as revealed by $\mu$-Auger spectroscopy. ${ }^{13}$ Since we carried out the EM experiment again in UHV at $80 \mathrm{~K}$, we expect that the central part close to the gap is completely free of contaminants.

So far, we have demonstrated how UHV compatible and extremely flat contacts in the nanometer-range can be generated by EM. However, these gaps can be closed again simply by thermal annealing to $300 \mathrm{~K}$. Electrical data during cycles of opening and closing the structure by EM and thermal annealing, respectively, are shown in Fig. 3(a) by $I V$ curves. The inset shows a magnification of the open state (note the

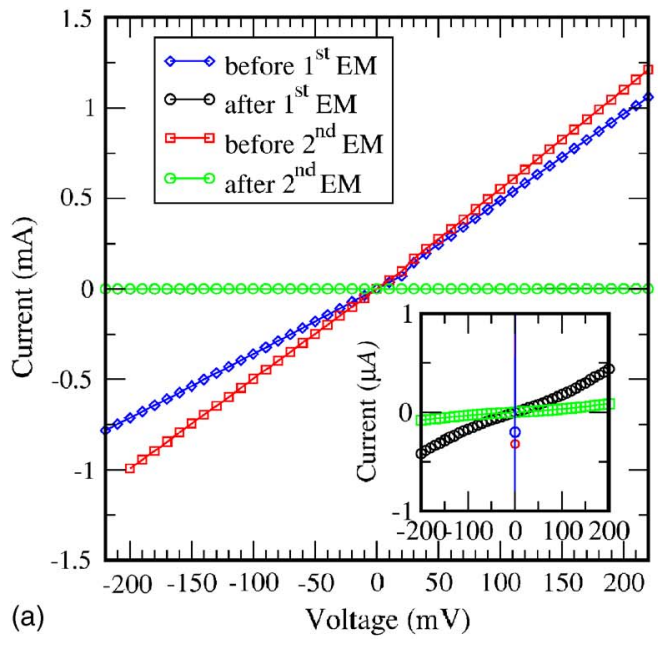

(a)

Voltage $(\mathrm{mV})$

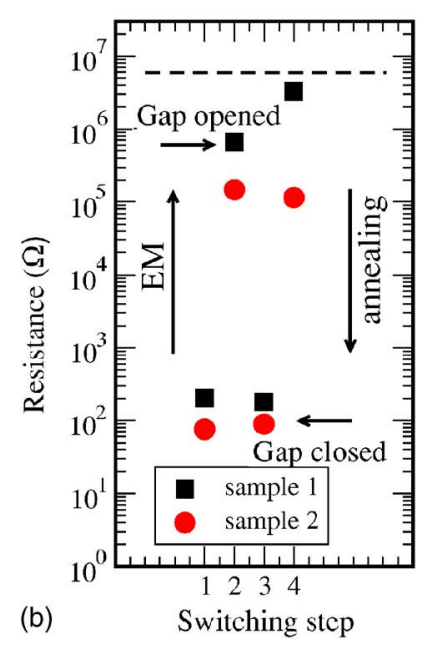

FIG. 3. (Color online) Reversible switching between an open and a closed state of the gap induced by EM and annealing, respectively. (a) shows (linear) $I V$ characteristics, indicating either the metallic or tunneling regime. The typical (zero bias) resistances of the two states are in the order of $0.1 \mathrm{k} \Omega$ (closed) and $100 \mathrm{k} \Omega-10 \mathrm{M} \Omega$ (open) as shown for two different samples in (b). 

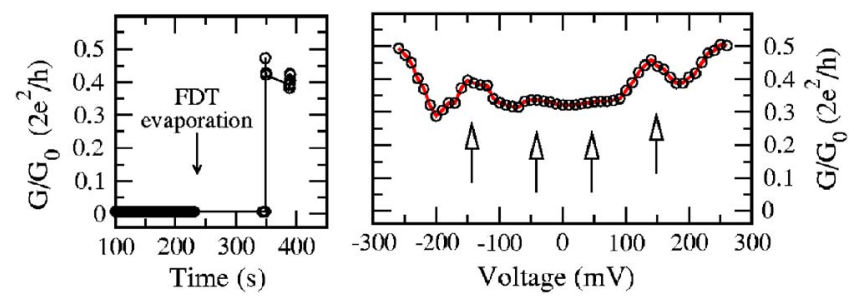

FIG. 4. (Color online) Change of the normalized conductance (left) after evaporation of FDT. The $d I / d V$ curve (right) shows molecular induced features around 50 and $150 \mathrm{meV}$.

different scales). The $I V$ curves are not fully symmetric possibly due to different contact resistances of the macroscopic leads (annealing reduced the deviation from the linear curves). Although the open/close behavior was reproducible, the absolute values scattered due to too slow regulation during the late stage of EM. Especially, the tunneling resistance of the gaps varied by up to one order of magnitude. A summary of this switching process for two different samples is shown in Fig. 3(b). This experimental finding proves that the $\mathrm{Ag}$ layers are closed and wet the Si surface. Furthermore, the property of opening and closing the gap can be useful for varying gap sizes.

Depending on the substrate temperature, the contacts withstand voltages up to $1.5 \mathrm{~V}$; i.e., the electric fields should not exceed $5 \times 10^{6} \mathrm{~V} / \mathrm{cm}$. At higher values of the electric field, discharge effects occur and, together with local heating and annealing, lead to formation of long crystalline Ag wires. As we have found by SEM measurements, this effect can be used to grow crystalline $\mathrm{Ag}$ wires as along as $0.2 \mathrm{~mm}(!)$ and $30 \mathrm{~nm}$ in diameter. Details will be reported elsewhere. ${ }^{14}$

Finally, and in order to demonstrate the functionality of our epitaxial contacts, ferrocenedithiol (FDT) molecules $\left(\mathrm{C}_{10} \mathrm{H}_{12} \mathrm{~S}_{2} \mathrm{Fe}\right.$, length $9 \AA$ ) (Ref. 15) were evaporated thermally out of a ceramic crucible onto the contact structure at a surface temperature close to $80 \mathrm{~K}$, as controlled by a $\mathrm{Ni} / \mathrm{NiCr}$ during evaporation. In the electrical experiment shown in Fig. 4, a gap of less than $1 \mathrm{~nm}$ with an initial resistance of $R=650 \mathrm{k} \Omega$ (at $V=0.3 \mathrm{~V}$ ) was used. Up to $250 \mathrm{~s}$ evaporation time, no changes of $R$ were observed. At this point, the molecular flux was turned off. Several seconds later, the conductance switched suddenly by almost an order of magnitude to $0.4 G_{0}\left(G_{0}=77.5 \mu S\right)$, indicating a field induced capturing of the molecule in between the contacts. This value remained stable with time over several hours. This resistance at zero bias of around $40 \mathrm{k} \Omega$ is in between the values which were measured for the open/closed gap configuration. Whereas the $I V$ curves for the open gaps were always found to be structureless, a clear molecule induced signature is now visible in the $I V$ curves around 50 and $150 \mathrm{meV}$. These curves resemble those obtained recently as enhanced transmission for ferrocene-oligophenylethynyldithiol placed in between Au contacts. ${ }^{16}$ In addition, their measured conductance values are very similiar to ours and seem to be characteristic of ferrocene and its derivatives. We note that the noise level for voltages above $\pm 200 \mathrm{mV}$ was quite high, indicating configurational fluctuations, which may be partly field (or current) induced. Therefore, for ongoing experiments the evaporation process will be transferred to our variable temperature STM, which can be operated with a $\mathrm{He}$ cryostat.

In summary, we have shown a viable process to generate not only ultrasmall, but also ultrathin molecular contacts which are produced by a combination of feedback-controlled EM applied to the ultrathin $(2-5 \mathrm{~nm}) \mathrm{Ag}$ nanostructures, grown on $\mathrm{Si}(100)$ surface as a wetting layer, and electron beam lithography. Due to their small thickness, these contacts can be imaged also within the gap with a STM, so that information about the local configurations of contacts and the molecules in between can be obtained. The gaps are chemically clean, and can be reversibly closed again by annealing to room temperature. In addition, our combination of STM with lateral conductance measurements showed that contacts with spacings in the range between several nanometers down to the subnanometer region were produced, which are suitable for capturing single molecules in between.

The authors greatfully acknowledge the financial support by the VolkswagenStiftung (I/79 310).

${ }^{1}$ H. Park, A. K. L. Lim, J. Park, A. P. Alivisatos, and P. L. McEuen, Appl. Phys. Lett. 75, 301 (1999).

${ }^{2}$ M. A. Reed, C. Zhou, C. J. Muller, T. P. Burgin, and J. M. Tour, Science 278, 252 (1997)

${ }^{3}$ R. H. M. Smit, Y. Noat, C. Untiedt, N. D. Lang, M. C. van Hemert, and J. M. van Ruitenbeek, Nature (London) 419, 906 (2002).

${ }^{4}$ T. Boehler, G. Grebing, A. Mayer-Gindler, H. von Loehneysen, and E. Scheer, Nanotechnology 15, S465 (2004).

${ }^{5}$ R. Krahne, I. Bar-Joseph, T. Dadosh, and J. Sperling, Appl. Phys. Lett. 81, 730 (2002).

${ }^{6}$ M. D. Fischbein and Marija Drndic, Appl. Phys. Lett. 88, 063116 (2006).

${ }^{7}$ E. Braun, Y. Eichen, U. Sivan, and G. Ben-Yoseph, Nature (London) 391, 775 (1998).

${ }^{8}$ R. Liu, S.-H. Ke, W. Yang, and H. U. Baranger, J. Chem. Phys. 124, 24718 (2006).

${ }^{9}$ K.-H. Müller, Phys. Rev. B 73, 45403 (2006).

${ }^{10}$ M. Horn-von Hoegen, Th. Schmidt, M. Henzler, G. Meyer, D. Wienau, and K. H. Rieder, Surf. Sci. 331-333, 575 (1995).

${ }^{11}$ H. H. Weitering, J. P. Sullivan, R. J. Carolissen, R. PAcrez-Sandoz, W. R. Graham, and R. T. Tung, J. Appl. Phys. 79, 7820 (1996).

${ }^{12}$ C. Kergueris, J.-P. Bourgoin, S. Palacib, D. Esteve, C. Urbina, M. Magoga, and C. Joachim, Phys. Rev. B 59, 12505 (1999); K. Hansen and M. Brandbyge, J. Appl. Phys. 95, 3582 (2004).

${ }^{13}$ For details about the Auger electron spectroscopy (AES) apparatus see V. Zielasek, T. Block, and H. Pfnür, Rev. Adv. Mater. Sci. 8, 1 (2004).

${ }^{14} \mathrm{G}$. Gardinowski and C. Tegenkamp (unpublished).

${ }^{15} \mathrm{M}$. Vollmann and H. Butenschön's synthesis of ferrocenedithiol according to J. J. Bishop, A. Davison, M. L. Katcher, D. W. Lichtenberg, R. E. Merill, and J. C. Smart, J. Organomet. Chem. 27, 241 (1971) using improved parameters..

${ }^{16}$ S. A. Getty, C. Engtrakul, L. Wang, R. Liu, S.-H. Ke, H. U. Baranger, W. Yang, M. S. Fuhrer, and L. R. Sita, Phys. Rev. B 71, 241401(R) (2005). 